Original Research Paper

\title{
Optimization of Raw Materials for Preparation of Transparent Soil Based on Analytic Hierarchy Process
}

\author{
${ }^{1,2}$ Jianjun Liu, ${ }^{1,2}$ Yao Wang, ${ }^{1,2}$ Rui Song and ${ }^{3}$ Min Ye \\ ${ }^{1}$ State Key Laboratory of Oil and Gas Reservoir Geology and Exploitation, \\ Southwest Petroleum University, Chengdu 610500, China \\ ${ }^{2}$ School of Geoscience and Technology, Southwest Petroleum University, Chengdu 610500, China \\ ${ }^{3}$ Urban and Rural Planning and Design Research Institute Ltd Co of Suining, Suining 629000, China
}

\author{
Article history \\ Received: 08-01-2017 \\ Revised: 16-01-2017 \\ Accepted: 25-02-2017 \\ Corresponsding author: \\ Yao Wang \\ State Key Laboratory of Oil \\ and Gas Reservoir Geology and \\ Exploitation, Southwest \\ Petroleum University, Chengdu \\ 610500 , China and \\ School of Geoscience and \\ Technology, Southwest \\ Petroleum University, Chengdu \\ 610500, China \\ Tel: +8615982260369 \\ Fax: +8602883035158 \\ Email:542385778@qq.com
}

\begin{abstract}
The application of man-made transparent soil has greatly contributed to the development of the visual research in physical model test. Comparing to the traditional model test, the transparent soil has advantages with respect to transparency, visualization, lower cost and utility et al. However, the transparency of material itself seriously restricted the effect of the results during the model text. This paper took the transparency as the evaluation standard, based on the Analytic Hierarchy Process (AHP) to make clear the factors on transparency of transparent soil which are: Purity and transparency of materials, matching degree of refractive index, size distribution of aggregate, air content and loadingunloading rate while consolidation and taking the commonly used combinations of raw materials as the example, the optimal combination was obtained. The work described in this study can provide a certain theoretical guidance for selection of raw materials and to improve the transparency during the preparation of transparent soil.
\end{abstract}

Keywords: Transparent Soil, Model Experiment, Optimization, Analytic Hierarchy Process

\section{Introduction}

Physical model test is a test method to obtain the regularity of the model prototype which is based on the similarity theory. According to a certain proportion a similar model of prototype will be built in the test, then through the indoor test or in situ test to obtain some change laws of model parameters (such as stress, strain and seepage), finally pushed these parameters back to prototype (Wang and Wang, 1992; Chai et al., 2014). It plays an important role in the study of geotechnical engineering. However, due to the opacity of the material in the traditional physical model test, the measurement of the deformation focused on the continuous deformation at the surface or boundary of the model. The measurement of stress is also depended on the preembedded sensors and it can only obtain the single monitoring result. However, it is still difficult to obtain the information about the damage time node of the internal structure, the formation and revolution law of the fractures and the concrete seepage process during the model test (Shen, 2011; Wu, 2006; Li, 2003).
The limitation in traditional physical model test has greatly restricted the further development of the model test. In order to realize the visual observation during the model test, Iskander et al. (2002a; 2002b) proposed a new method of physical model test which is based on the transparent soil, combining with optical observation method and digital image processing technique. It has made a great breakthrough on the visual study of the physical model test.

The application of transparent soil in the physical model test has greatly promoted the visual study of model test. However, with a comprehensive analysis of the existing research results (Iskander et al., 1993; Ezzein and Bathurst, 2011; 2014), the transparency of the transparent materials used in the test has become a key factor that restricts the results of the model test and it decided the results quality of the model test to a great extent. So it is very necessary to develop the study on the influence factors of transparency. This paper summarized the preparation process of transparent soil and the main factors affecting the transparency of the sample were proposed and the 
weights of the factors were determined based on the AHP and the suggestions to promote the transparency of the sample were given qualitatively and quantitatively and the matters needing attention were pointed out during the preparation process. At the same time, aiming at the common used combination of raw materials in preparation of transparent soil, the weights of different raw materials affecting the transparency were obtained through the calculation based on the AHP, thus the optimal combination of raw material was determined.

\section{Physical Model Test Based on Transparent Soil}

Transparent soil is synthesized by two or more than two kinds of materials which have similar Refractive Index (RI) according to a certain proportion and it has the similar characteristics from the perspective of strength and deformation with the natural rock-soil, thus it can be used to simulate the natural rock-soil (Liu, 2003). The model test based on transparent rock soil material, as the name suggests is the application of transparent soil in traditional model test, combining the optical observation method and digital image processing technique, thus realized the visual observation of the damage of internal structure and seepage process during the test. Iskander and Liu (2010; Iskander, 2010) developed a series device of model test for the use of transparent soil (schematic diagram of device as shown in Fig. 1). After many years' improvement and development (Fernandez et al.,
2011; Sui et al., 2011), this technology has become more mature and has made a certain achievements from the perspective of the interaction mechanism of internal structure in the rock-soil body, the analysis of seepage field and the 3D deformation.

\section{Preparation Technology of Transparent Soil}

Since (Mannheimer, 1990), in order to study the flow problems of non-Newtonian fluid, Mannheimer invented a transparent slurry. After many years' improvement and experimental study of many scholars, it has formed a set of perfect preparation process of transparent soil (Iskander et al., 2002a; Zhang, 2014; Xu 2011; Li and Lin, 2015).

Aiming at the different test purposes and methods, there may be something different in the process, but with a comprehensive analysis of exiting study, the preparation process can be summarized in the following steps which are shown in Fig. 2.

\section{Factors on Transparency of the Sample}

With the analysis of sample preparation process, it shows that there are 4 points needing to be considered during the preparation process: (1) The determination of aggregate distribution and matching of pore fluid; (2) Proportioning experiment to determine the best ratio of aggregate and pore fluid; (3) Mix aggregate and pore fluid together while stirred continuously and vacuum treatment to exhaust air at the same time; (4) Loading and unloading step by step to consolidate the sample.

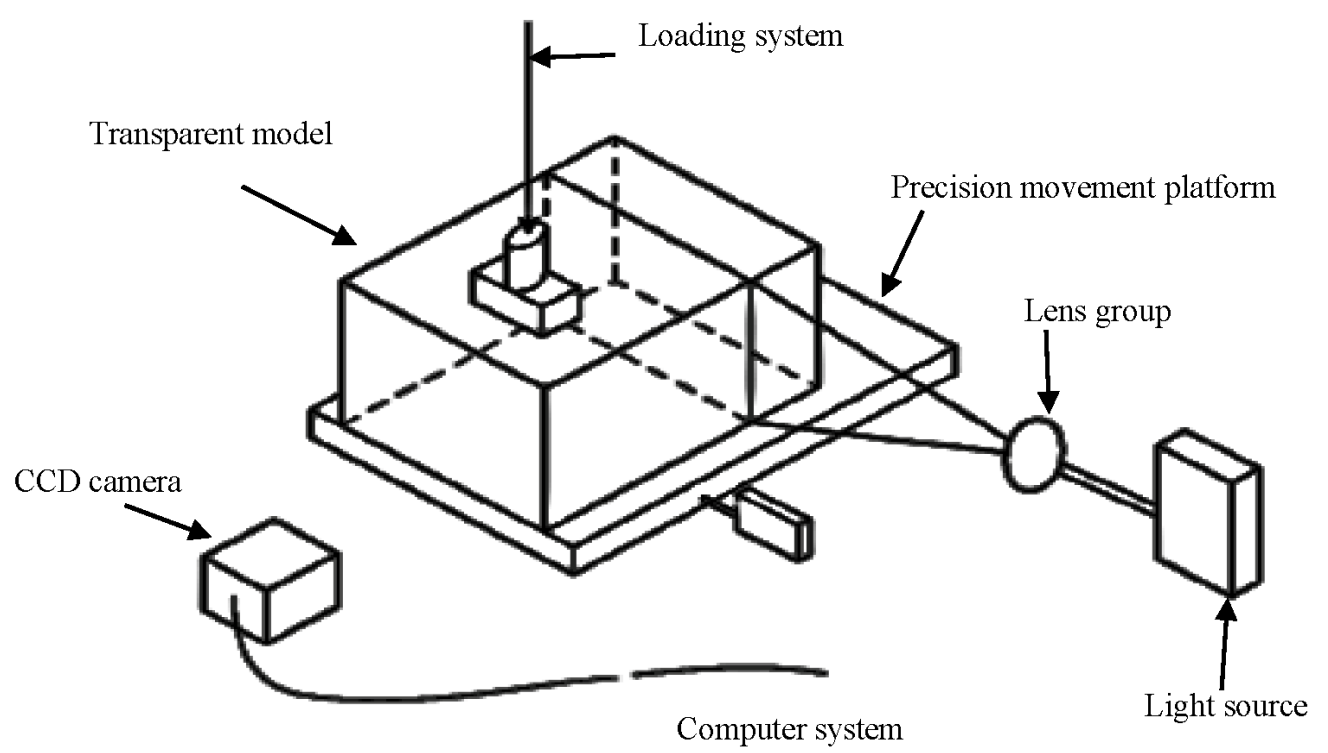

Fig. 1. Schematic diagram of model test device 


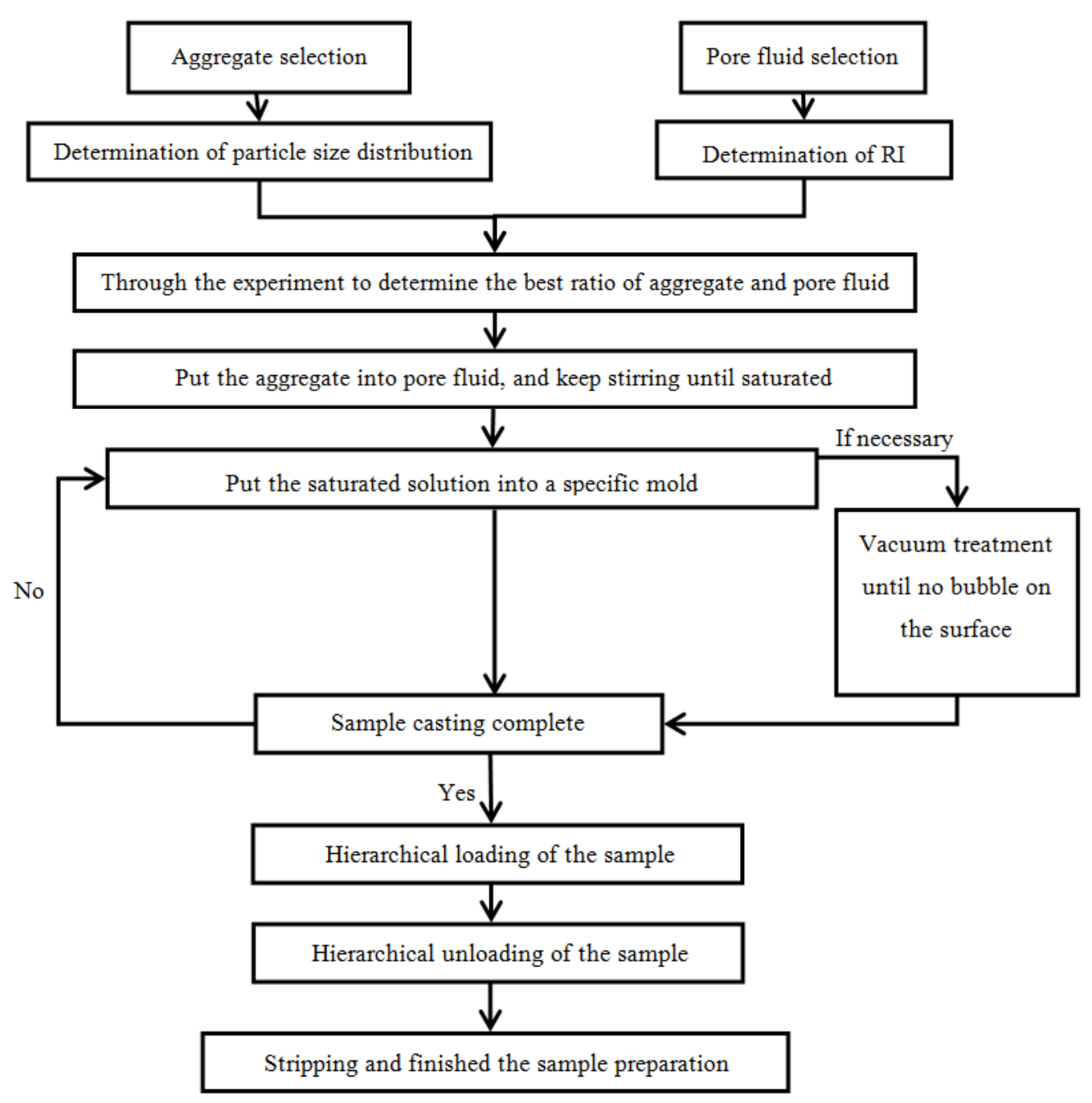

Fig. 2. Flow chart of sample preparation

Aiming at the 4 points where need to pay attention, five influence factors on transparency of the sample can be induced.

For step (1): The determination of aggregate distribution is to ensure that the sample has certain intensity under the condition of meeting the requirements of transparency. Because when the aggregate size is bigger, the transparent material sample has a higher transparency, however the sample will has a lower intensity, because the mixture is prone to segregation due to the large size of aggregate, even when the particle size is large to a certain degree that we can't obtain the figurate sample due to the exhaust speed of pore fluid is too quick while consolidation. On the contrary, when the aggregate size is too small, the sample will have a larger intensity, but poor transparency, even when the particle size is small to a certain degree that vacuum treatment can't exhaust the air in the mixture; The selection of pore fluid is in order to ensure the matching of RI between aggregate and pore fluid; At the same time, this step should also ensure the purity and transparency of the materials themselves.

For step (2): The best experimental ratio of aggregate and pore fluid was determined by the ratio experiment and also to ensure the best transparency of the prepared samples.

For step (3): Continuously stirring during mixing aggregate and pore fluid and vacuum treatment after mixing are both to exhaust air in the mixture, so that eliminate the effect of air bubbles on transparency of samples.

For step (4): The control of rate of loading and unloading step by step is also to avoid the situation that the pore fluid is too fast to be discharged while loading too fast, thus affecting the overall transparency of the sample.

Through the above analysis, it can be concluded that the main influence factors on transparency of transparent 
soil are: The purity and transparency of raw materials themselves, the matching degree of RI between aggregate and pore fluid, the distribution of aggregate size, the air content in the mixture and the rate of loading and unloading step by step.

\section{Common Used Raw Material Combination}

Through consulting the relevant literatures (Kong et al., 2016; Li and Lin, 2015; Iskander, 2010; Iskander et al., 2002a; 2002b; Ezzein and Bathurst, 2011; Sui et al., 2011; Zhang et al., 2014), the currently common used aggregate are: Amorphous silica powder and silica gel, the pore fluid: Mineral oil and calcium bromide solution. The different combinations of aggregate and pore fluid are following four types: (i) Amorphous silica powder and Mineral oil; (ii) Silica gel and Mineral oil; (iii) Amorphous silica powder and Calcium bromide solution; (iv) Silica gel and Calcium bromide solution.

The amorphous silica powder, commonly known as "precipitated silica", the particles have two types: Transparent and opaque, but the macro performance is white due to the diffraction and refraction of the light. The average particle size of the particle distributes in the range of 1.4 175 $\mu \mathrm{m}$, the RI between $1.41 \sim 1.46$ and the property is relatively high purity, low stress, high moisture resistance and so on.

The silica gel is the colloidal form of silica which presents amorphous, porous and part hydrophilic. The average particle size of the particle distributes in the range of $0.5 \sim 5 \mathrm{~mm}$ and has the same RI with amorphous silica powder, but has a higher transparency than amorphous silica powder in the same pore fluid.

The mineral oil is synthesized by mixing two kinds of non-miscible fluid which RI is larger and smaller than the matching aggregate, respectively. The common used fluid types are: Paraffin, normal-twelve alkyl, normal-thirteen alkyl or white mineral oil. The RI is generally ranged from $1.42 \sim 1.46$ after mixing and the viscosity is about $0.005 \mathrm{pa} \cdot \mathrm{s}$.

The calcium bromide solution is a brine mixture after dissolving calcium bromide in water in a certain proportion. The RI of calcium bromide solution has a larger rang with the change of concentration which is between $1.34 \sim 1.48$ and the viscosity is about $0.0036 \mathrm{pa} \cdot \mathrm{s}$.

\section{Quantitative Evaluation of Factors and Optimization of Raw Material Combination Based on AHP}

\section{Analytic Hierarchy Process (AHP)}

The Analytic Hierarchy Process (AHP) is a multiobjective decision analysis method which combines the qualitative and quantitative analysis. By using this method, the complex problem is decomposed into a plurality of layers and a number of factors, then conducts pairwise alignments among the multiple factors respectively and makes comparative judgments of its importance, constructing the judgment matrix and then the weights of different importance degree of factors are obtained through relevant calculation and finally conducts a consistency test to determine the rationality of the calculation. The method has the advantages of systematicness, flexible and simple calculation (Deng et al., 2012; Saaty, 1980).

Through consulting the relevant literatures (Saaty, 1980), the basic steps of AHP can be summarized as follows:

- Aiming at specific problems, establishing the corresponding analytic hierarchy model (the target layer, criterion layer and scheme layer)

- Constructing judgment matrix of each influence factor of the corresponding criterion layer

- Solving the judgment matrix and get the weight of the importance degree of each influence factor

- Conducting consistency test to judge the rationality of the calculation

\section{Weight Calculation of Factors}

\section{Establishment of the Analytic Hierarchy Model}

Aiming at the analysis objects of this paper the influence factors on transparency of transparent soil and the optimization of combination of raw materials, combined with the five factors identified above and different material combination, we could establish the analytic hierarchy model according to the calculation steps of AHP. First, we could define the transparency of transparent soil as the target layer which was expressed as symbol T. Second we could define the five influence factors as the criterion layer which was expressed as symbol $\mathrm{F}_{1}, \mathrm{~F}_{2}, \mathrm{~F}_{3}, \mathrm{~F}_{4}$ and $\mathrm{F}_{5}$, respectively and then the four different combinations of raw materials could be defined as the scheme layer which was expressed as symbol $\mathrm{P}_{1}, \mathrm{P}_{2}, \mathrm{P}_{3}$ and $\mathrm{P}_{4}$, respectively. Finally we could establish the following analytic hierarchy model, as shown in Fig. 3.

\section{Establishment of Judgment Matrix}

We can use the 1-9 scale proposed by Saaty (1980) to compare the influence degree of two different kinds of factors $F_{i}$ and $F_{j}$ for the upper layer $T$ while constructing the judgment matrix A. And the range of judgment matrix A's elements $a_{i j}$ is $1 \sim 9$ or the reciprocal number $1 \sim 1 / 9$ (Saaty, 1980). The judgment matrix is established based on the scale meaning which is shown as the following Table 1. 
According to the analysis of the preparation process of transparent soil, we could conduct pairwise comparisons to the importance degree of five influence factors on transparency. Then combining with judgment matrix scale, we could establish the judgment matrix $A$ of the target layer to criterion layer based on the comparative scale shown in Table 2.

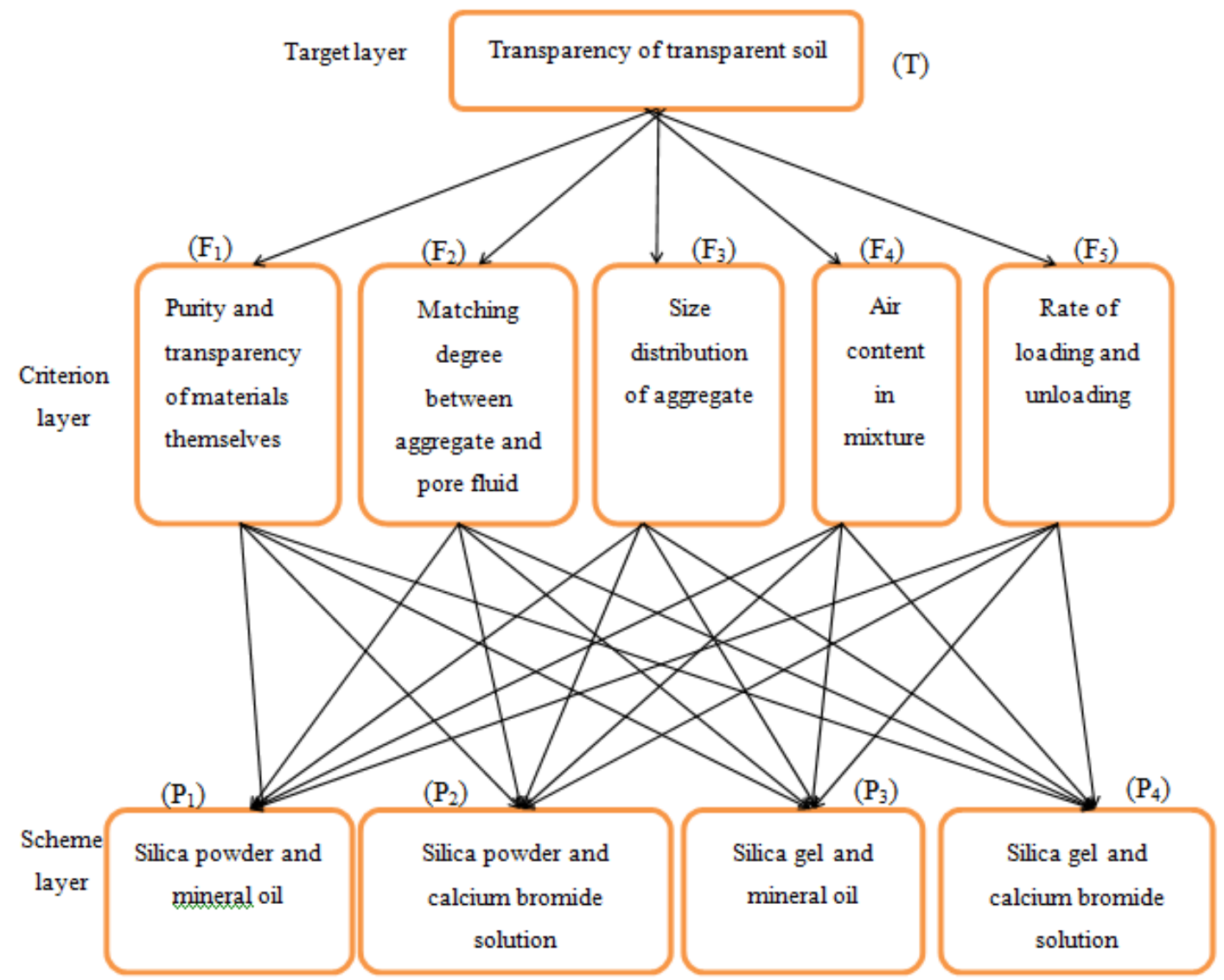

Fig. 3. Schematic diagram of analytic hierarchy model

Table 1. Scale definition of the judgment matrix

\begin{tabular}{ll}
\hline Scale $\mathrm{a}_{\mathrm{ij}}$ & Definition \\
\hline 1 & $\mathrm{~F}_{\mathrm{i}}$ has the same influence with $\mathrm{F}_{\mathrm{j}}$ \\
3 & $\mathrm{~F}_{\mathrm{i}}$ is a little stronger than $\mathrm{F}_{\mathrm{j}}$ \\
7 & $\mathrm{~F}_{\mathrm{i}}$ is stronger than $\mathrm{F}_{\mathrm{j}}$ \\
9 & $\mathrm{~F}_{\mathrm{i}}$ is much stronger than $\mathrm{F}_{\mathrm{j}}$ \\
$2,4,6,8$ & $\mathrm{~F}_{\mathrm{i}}$ is absolute stronger than $\mathrm{F}_{\mathrm{j}}$ \\
$1,1 / 2, \cdots 1 / 9$ & The influence ratio of $\mathrm{F}_{\mathrm{i}}$ and $\mathrm{F}_{\mathrm{j}}$ between the above adjacent levels \\
\hline
\end{tabular}

Table 2. Assignment of the comparative scale of judgment matrix

\begin{tabular}{llllll}
\hline T & F1 & F2 & F3 & F4 & F5 \\
\hline F1 & 1 & 1 & 3 & 5 & 7 \\
F2 & 1 & 1 & 3 & 5 & 7 \\
F3 & $1 / 3$ & $1 / 3$ & 1 & 3 & 5 \\
F4 & $1 / 5$ & $1 / 5$ & $1 / 3$ & 1 & 3 \\
F5 & $1 / 7$ & $1 / 7$ & $1 / 5$ & $1 / 3$ & 1 \\
\hline
\end{tabular}


As shown in Table 2, the matrix A can be obtained as follows:

$$
A=\left[\begin{array}{ccccc}
1 & 1 & 3 & 5 & 7 \\
1 & 1 & 3 & 5 & 7 \\
1 / 3 & 1 / 3 & 1 & 3 & 5 \\
1 / 5 & 1 / 5 & 1 / 3 & 1 & 3 \\
1 / 7 & 1 / 7 & 1 / 5 & 1 / 3 & 1
\end{array}\right]
$$

\section{Weight Calculation}

The summation method is a simple and practical algorithm to calculate the weight based on AHP and the calculation steps are shown as follows:

First, normalization each column vector of $A$ to obtain $A_{1}\left(\varpi_{i j}=a_{i j} / \sum_{i=1}^{n} a_{i j}\right)$ :

$$
A_{1}=\left[\begin{array}{lllll}
0.3737 & 0.3737 & 0.3982 & 0.3488 & 0.3043 \\
0.3737 & 0.3737 & 0.3982 & 0.3488 & 0.3043 \\
0.1246 & 0.1246 & 0.1327 & 0.2093 & 0.2174 \\
0.0747 & 0.0747 & 0.0442 & 0.0698 & 0.1304 \\
0.0534 & 0.0534 & 0.0265 & 0.0233 & 0.0435
\end{array}\right]
$$

Second, summation $A_{1}$ according to the line respectively to obtain $A 2\left(\varpi_{i}=\sum_{i=1}^{n} \varpi_{i j}\right)$ :

$$
A_{2}\left[\begin{array}{l}
1.7987 \\
1.7987 \\
0.8086 \\
0.3938 \\
0.2001
\end{array}\right]
$$

Finally, normalization $A_{2}$ according to the column to obtain $A_{3}$ and that is the weight vector and the each element of the vector is the weight of each factor:

$$
A_{3}=\left[\begin{array}{l}
0.3597 \\
0.3597 \\
0.1617 \\
0.0788 \\
0.0400
\end{array}\right]
$$

According to the calculation results, we can know that the weight of the five influence factors on transparency of transparent soil are: The purity and transparency of materials themselves (0.3597), the matching degree of RI between aggregate and pore fluid (0.3597), the size distribution of aggregate $(0.1617)$, the air content in mixture $(0.0788)$ and the rate of loading and unloading step by step (0.04), respectively.

In addition, Saaty (1980) had proposed that the eigenvector corresponding to the maximum eigenvalue of the matrix A can be conducted to normalization processing and the results after processing can be used as the weight corresponding to the importance degree of influence factor. In this study, we can use the function "eig" to calculate the eigenvector corresponding to the eigenvalue through Matlab and the implementation procedure is shown as follows:

- Clear \%Clear variable in workspace

- $\quad$ Clc \%Clear command window

- $[\mathrm{V}, \mathrm{D}]=\operatorname{eig}(\mathrm{A}) \% \mathrm{~V}$ is eigenvector matrix and $\mathrm{D}$ is eigenvalue matrix

- $\quad$ End \%Finish of program

The maximum eigenvalue is $\lambda_{\max }=5.1361$ and the eigenvector matrix $V$ corresponding to the maximum eigenvalue is shown as follows:

$$
V=\left[\begin{array}{l}
0.6663 \\
0.6663 \\
0.2957 \\
0.1402 \\
0.0716
\end{array}\right]
$$

Normalization processing of $\mathrm{V}$ can obtain the weight vector $V_{1}$ of each influence factor:

$$
V_{1}=\left[\begin{array}{l}
0.3621 \\
0.3621 \\
0.1607 \\
0.0762 \\
0.0389
\end{array}\right]
$$

By comparing the $A_{3}$ and $V_{1}$, it can be found that the weight error calculation through the two different kinds of methods is not more than $1 \%$, so we can confirm that the results obtained through the two above methods are both correct.

\section{Consistency Test}

Because the judgment matrix $A$ is established based on the qualitative analysis of the preparation process of transparent soil and the comparison scale, so there may be an internal logical contradiction, thus the calculation results of the weights need to conduct further consistency test.

The consistency ratio $C R$ will be introduced into the consistency test to check the judgment matrix. When the 
$C R<0.1$, that is the inconsistency degree of the judgment matrix A within the admissible range and the formula of consistency ratio $C R$ is shown as following Equation 1:

$C R=\frac{C I}{R I}$

In the formula: $C I$ is the consistency index; $R I$ is random consistency index and $C I$ is given through the following Equation 2:

$C I=\frac{\lambda_{\max }-n}{n-1}$

In the formula: $\lambda_{\max }$ is the maximum eigenvalue of the matrix $A ; n$ is the order number of the matrix $\mathrm{A}$. $R I$ is given through the following Table 3 .

According to the above calculation results, the consistency index can be obtained $\mathrm{CI}=0.034$, the $\mathrm{RI}=$ 1.12 through looking up the Table 3 , so the consistency ratio $C R$ is 0.0304 , that is $C R<0.1$. So, the inconsistency degree of the judgment matrix A within the admissible range and calculation results are reasonable.

\section{Optimization of Raw Material Combinations}

\section{Weight Calculation of Scheme Layer to Criterion Layer}

According to the construction process of above judgment matrix, with a comprehensive analysis of relationship between the characteristics of raw materials and each influence factor, combining with the comparative scale, we can establish the judgment matrix $F_{i}$ of criterion layer to scheme which is shown as follows:

$$
F_{1}=\left[\begin{array}{cccc}
1 & 1 / 3 & 1 / 5 & 1 / 3 \\
3 & 1 & 1 / 3 & 1 \\
5 & 3 & 1 & 3 \\
3 & 1 & 1 / 3 & 1
\end{array}\right]
$$

$$
\begin{gathered}
F_{2}=\left[\begin{array}{cccc}
1 & 3 & 1 & 3 \\
1 / 3 & 1 & 1 / 3 & 1 \\
1 & 3 & 1 & 3 \\
1 / 3 & 1 & 1 / 3 & 1
\end{array}\right] \\
F_{3}=\left[\begin{array}{cccc}
1 & 1 & 3 & 3 \\
1 & 1 & 3 & 3 \\
1 / 3 & 1 / 3 & 1 & 1 \\
1 / 3 & 1 / 3 & 1 & 1
\end{array}\right] \\
F_{4}=\left[\begin{array}{cccc}
1 & 3 & 1 / 3 & 1 / 3 \\
1 / 3 & 1 & 1 / 5 & 1 / 3 \\
3 & 5 & 1 & 3 \\
3 & 3 & 1 / 3 & 1
\end{array}\right] \\
F_{5}=\left[\begin{array}{cccc}
1 & 1 / 3 & 1 / 3 & 1 / 5 \\
3 & 1 & 1 & 1 / 3 \\
3 & 1 & 1 & 1 / 3 \\
5 & 3 & 3 & 1
\end{array}\right]
\end{gathered}
$$

Using the above mentioned method to solve the each judgment matrix, the weight $\omega^{(3)}$ of scheme layer to criterion layer can be obtained respectively. At the same time, we can also calculate $C I$ and $C R$, so as to carry out the consistency test and the Calculation results are shown in the following Table 4.

It can be seen from the calculation results of Table 4 that the results of weight calculation all meet the requirement of consistency.

\section{Combined Weight Calculation}

Using the weight calculation results of the criterion layer to target layer and the scheme layer to criterion layer, the combined weight of the scheme layer to target layer can be obtained, so as to realize the combination optimization of raw materials based on transparency.

Table 3. The values of random consistency index $R I$

\begin{tabular}{llllllllllll}
\hline $\mathrm{n}$ & 1 & 2 & 3 & 4 & 5 & 6 & 7 & 8 & 9 & 10 & 11 \\
\hline$R I$ & 0 & 0 & 0.58 & 0.9 & 1.12 & 1.24 & 1.32 & 1.41 & 1.45 & 1.49 & 1.51 \\
\hline
\end{tabular}

Table 4. The weight calculation results of the combinations of the scheme layer

\begin{tabular}{llllll}
\hline $\mathrm{F}_{\mathrm{i}}$ & 1 & 2 & 3 & 4 & 5 \\
\hline$\omega^{(3)}$ & 0.0781 & 0.375 & 0.375 & 0.1512 & 0.0781 \\
& 0.1998 & 0.125 & 0.375 & 0.0752 & 0.1998 \\
& 0.5222 & 0.375 & 0.125 & 0.5083 & 0.1998 \\
& 0.1998 & 0.125 & 0.125 & 0.2653 & 0.5222 \\
$C I^{(2)}$ & 0.0145 & 0.000 & 0.000 & 0.0660 & 0.0145 \\
$C I^{(3)}$ & 0.0161 & 0.000 & 0.000 & 0.0733 & 0.0161 \\
\hline
\end{tabular}


The weight $\omega^{(2)}$ is the calculation result of the criterion layer to target layer. The weight $\omega^{(3)}$ is the calculation result of the scheme layer to criterion layer. The weight $\omega^{(1)}$ of the scheme layer to target layer can be obtained through Equation 3:

$$
\omega_{i}^{(1)}=\omega^{(2)} * \omega_{i}^{(3)}(\mathrm{i}=1 \sim 5)
$$

The calculation of first combination is shown as follows:

$$
\begin{aligned}
& \omega_{1}^{(1)}=0.3597 * 0.0787+0.3597 * 0.375+ \\
& 0.1617 * 0.375+0.0788 * 0.1512+0.04 * 0.0781
\end{aligned}
$$

$\omega_{1}^{(1)}=0.2389$. In the same way, the combined weights of other three combinations can be obtained $\omega_{2}^{(1)}$ $=0.1914, \omega_{3}^{(1)}=0.3915$ and $\omega_{4}^{(1)}=0.1782$, respectively.

And then conducting combined consistency test through Equation 4:

$$
C R=\frac{\sum_{i=1}^{n} \omega_{i}^{(2)} C I_{i}}{\sum_{i=1}^{n} \omega_{i}^{(2)} R I_{i}}
$$

Through calculation, the combined consistency ratio $C R=0.012$ can be obtained, also the combined consistency ratio $C R<0.1$, so the combined consistency test is also qualified.

Through the above calculation, the weights of the four different combinations to transparency are: Silica powder and mineral oil (0.2389); silica powder and calcium bromide solution (0.1914); silica gel and mineral oil (0.3915); silica gel and calcium bromide solution (0.1782), respectively, that is the transparent soil sample which is synthesized through silica gel and mineral oil has a relatively higher transparency.

\section{Analyses and Discussion of Results}

According to the above calculation results, the weights of importance degree of the five influence factors on transparency are respectively: The purity and transparency of materials themselves (0.3597), the matching degree of RI between aggregate and pore fluid $(0.3597)$, the size distribution of aggregate (0.1617), the air content in mixture $(0.0788)$ and the rate of loading and unloading step by step (0.04). That is the characteristics of materials themselves and the matching degree of RI between aggregate and pore fluid have the relatively larger influence on transparency. Secondly is the size distribution of aggregate. Then the air content in mixture and the rate of loading and unloading have the relatively smaller influence on transparency.

The influence of the four different raw materials on the transparency is: $0.2389,0.1914,0.3915$ and 0.1782 . In the combination of four kinds of raw materials, the raw material of silica gel and mineral oil can be used to make the transparent rock material with relatively good transparency in the condition that other preparation conditions are unchanged.

About the four different combinations of raw materials, in the case of other preparation conditions remain the same, the transparent soil sample synthesized through silica gel and mineral oil has a relatively higher transparency.

Through the above analysis, we can conclude that in order to obtain the sample with a relatively higher transparency. First, we can choose the silica gel and mineral oil as the raw material, then according to the preparation process, conducting qualitative analysis of the preparation process which may affect the transparency, finding out the specific influence factors, forming an intuitive realization. Second, the importance degree weights of the different influence factors on transparency can be obtained through the calculation, quantification the influence of each factor. Finally, qualitative analysis combined with quantitative analysis, which is more conducive to the improvement of the existing problems in preparation process and to make clear the matters needing attention in each procedure, reducing the influence on transparency.

\section{Conclusion}

In this study, five influence factors on transparency of transparent soil were proposed based on the summary of preparation process and the weights of influence factors were also obtained based on AHP. Make clear that the purity and transparency of materials themselves and the matching degree of RI between aggregate and pore fluid are the most important factors we need to pay attention. Secondly is the size distribution. Finally are the air content and rate of loading and unloading and these matters needing attention also indicate the improvement direction of preparation process.

At the same time, through the further study of the influence of different combination of raw materials on transparency, the influence weights of different combinations on transparency were obtained based on AHP. The results showed that the sample synthesized through silica gel and mineral oil has a relatively higher transparency among the currently common used raw materials. This method provides a new idea for the combination optimization of raw materials when the preparation of transparent soil. 


\section{Acknowledgement}

This paper is financially supported by Natural Science Foundation of China (Grant No. 51174170), National Science and Technology Major Project of China (Grant No. 2011ZX05013-006).

\section{Author's Contributions}

Jianjun Liu: Provided the writing idea of this manuscript and reviewed the full text after the completion of this manuscript.

Yao Wang: Completed the writing of this manuscript and the correction of the text.

Rui Song and Min Ye: Helped reviewed the final manuscript after the completion of the manuscript and provided the significant help during the writing of this paper.

\section{Ethics}

The authors declare that there is no conflict of interest and other ethical issues regarding the publication of this manuscript.

\section{References}

Chai, J., Z.L. Wang and Y. Li, 2014. Application analysis of physical model simulation method. J. Xi'an Univ. Sci. Technol., 34: 123-128.

Deng, X., J.M. Li and H.J. Zeng, 2012. Research on computation methods of AHP weight vector and its applications. Math. Pract. Theory, 42: 93-100.

Ezzein, F.M. and R.J. Bathurst, 2011. A transparent sand for geotechnical laboratory modeling. Geotech. Test. J., 34: 590-601. DOI: 10.1520/GTJ103808

Ezzein, F.M. and R.J. Bathurst, 2014. A new approach to evaluate soil-geosynthetic interaction using a novel pullout test apparatus and transparent granular soil. Geotextiles Geomenbranes, 42: 246-255. DOI: $10.1016 /$ j.geotexmem.2014.04.003

Fernandez, S.R., M. Iskander and K. Tabe, 2011. 3D contaminant flow imaging in transparent granular porous media. Geotechnique Lett., 1: 71-78. DOI: 10.1680/geolett.11.00027

Kong, G.Q., L.D. Zhou, Z.T. Wang and G. Yang, 2016. Shear modulus and damping ratios of transparent soil manufactured by fused quartz. Mater. Lett., 182: 257-259. DOI: 10.1016/j.matlet.2016.07.012

Iskander, M. and J.Y. Liu, 2010. Spatial deformation measurement using transparent soil. Geotech. Test. J., 33: 1-7. DOI: 10.1520/GTJ102745

Iskander, M., J. Lai, C. Oswald and R.J. Mannheimer, 1993. Development of transparent material to model the geotechnical properties of soils. Geotech. Test. J., 17: 425-433. DOI: 10.1520/GTJ10303J
Iskander, M., S. Sadek and J.Y. Liu, 2002a. Optical measurement of deformation using transparent silica gel to model sand. Int. J. Phys. Model Geomechan., 2: 13-26. DOI: 10.1680/ijpmg.2002.2.4.13

Iskander, M., J.Y. Liu and S. Sadek, 2002b. Transparent amorphous silica to model clay. J. Geotechnique Geoenviron. Eng., 128: 262-273.

DOI: 10.1061/(ASCE)1090-0241(2002)128:3(262)

Iskander, M., 2010. Modelling with Transparent Soils: Visualizing Soil Structure Interaction and Multi Phase Flow, Non-Intrusively. 1st Edn., SpringerVerlag, Berlin, Heidelberg, Germany, ISBN-10: 3642025013, pp: 335.

Li, Y.H. and Z.B. Lin, 2015. Innovative experimental method based on development of transparent rock mass materials for physical tests. Chinese J. Geotech. Eng., 37: 2030-2039. DOI: 10.11779/CJGE201511013

Li, Y.H., 2003. Study on digital photography-based deformation measurement technology and its application in geo-physical model experiment. $\mathrm{PhD}$ Thesis, Tongji University, Shanghai, China.

Liu, J.Y., 2003. Visualization of 3D deformations using transparent "soil" models. PhD Thesis, Polytechnic University, New York, USA.

Mannheimer, R., 1990. Slurries you can see through. Technol. Today, 3: 2-2.

Saaty, T.L., 1980. The Analytic Hierarchy Process. 1st Edn., McGraw-Hill Company, New York, America, ISBN-13: 9780070543713.

Shen, T., 2011. Development of geo-mechanic model experiment techniques. J. Yangtze River Scientific Res. Instit., 18: 32-36.

Sui, W.H., Y. Gao and J.Y. Liu, 2011. Status and prospect of transparent soil experimental technique. J. China Coal Society, 36: 577-582.

Wang, H.S. and X.K. Wang, 1992. Dictionary of Scientific Methods. 1st Edn., Zhejiang Education Press, Hangzhou, China, ISBN-10: 7-5338-0915-7/G.916.

Wu, M.X., 2006. Study on transparent synthetic sand and its' trixial test. PhD Thesis, Dalian University of Technology, Dalian, China.

$\mathrm{Xu}$, G.A., 2011. Research on the mechanism and evolution law of rock deformation and cracking around deep tunnels. PhD Thesis, China University of Mining and Technology, Xuzhou, China

Zhang, S.J., 2014. Study on the Development of Transparent Similar Materials for Rock Mass and Its Experimental Applications. MSc Thesis, China University of Mining and Technology, Xuzhou, China.

Zhang, Y.P., L. Li and S.Z. Wang, 2014. Experimental study on pore fluid for forming transparent soil. J. Zhejiang Univ., 48: 1828-1834.

DOI: $10.3785 /$ j.issn.1008-973X.2014.10.017 